\title{
Rendimiento académico y las dimensiones personal y contextual del aprendizaje socioemocional: evidencias de su asociación en estudiantes chilenos*
}

\author{
Academic Performance and Personal and Contextual Dimensions of \\ Socioemotional Learning: Evidence of its Association in Chilean Students
}

Recibido: enero 28 de 2013 | Revisado: mayo 26 de 2013 | Aceptado: octubre 3 de 2013

\author{
CHRISTIAN BERGER ** \\ Pilar Álamos *** \\ NeVA MILICIC **** \\ LIDIA AlCALAY
}

Pontificia Universidad Católica de Chile

doi:10.11144/Javeriana.UPSY13-2.radp

Para citar este artículo: Berger, C., Álamos, P., Milicic, N., \& Alcalay, L. (2014). Rendimiento académico y las dimensiones personal y contextual del aprendizaje socioemocional: Evidencias de su asociación en estudiantes chilenos. Universitas Psychologica, 13(2), 627-638. doi:10.11144/Javeriana. UPSY13-2.radp

Fondo Nacional de Desarrollo Científico y Tecnológico de Chile, FONDECYT, proyecto 1100391.

** Profesor Associado. Escuela de Psicología. Pontificia Universidad Católica de Chile. Correo electrónico: cberger@uc.cl

**** Correos electrónicos: pmalamos@uc.cl,nmilicic@uc.cl ,lalcalay@uc.cl

\section{RESUMEN}

El presente estudio respalda la creciente evidencia empírica sobre la relación entre la dimensión socioemocional y el rendimiento académico. Mediante análisis de correlación y regresión se testeó en estudiantes chilenos de educación básica la asociación entre el rendimiento académico y las dimensiones individual (autoestima y bienestar socioemocional) y contextual (percepción del clima social escolar) del aprendizaje socioemocional. Bienestar socioemocional y percepción del clima social escolar respecto a relaciones de pares y lugares de la escuela, mostraron una relación significativa con el aumento en el desempeño académico. Los hallazgos son discutidos en términos de sus implicancias para la práctica educativa y para futuras investigaciones. Palabras clave

Aprendizaje socioemocional, rendimiento académico, clima social escolar.

\footnotetext{
A B S T R A C T

This study supports the growing empirical evidence regarding the relationship between the socioemotional dimension and academic achievement. Through correlation and regression analyses the associations between individual (self-steem and socioemotional well-being) and contextual (perception of the school social climate) features of socioemotional learning and academic success in Chilean elementary students were tested. Socioemotional well-being and perception of school social climate with regards to peer relations and places at school were significantly related to improvements in academic achievement. Results are discussed in terms of their implications for educational practice and future research.

Keywords

Socioemotional learning, academic achievement, school social climate.
} 


\section{Introducción}

En los últimos años se ha generado importante evidencia respecto del impacto de la dimensión socioemocional en indicadores académicos a nivel internacional (Durlak, Weissberg, Dymnicki, Taylor \& Schellinger, 2011; Extremera \& Fernández-Berrocal, 2003). Estudios en distintos contextos han logrado relevar que desarrollar aspectos socioafectivos tiene impacto no solo en indicadores de bienestar y salud mental de los estudiantes, sino también favorece tanto su rendimiento académico como su autoconcepto (Berger, Alcalay, Torretti \& Milicic, 2011; Caso-Niebla \& Hernández-Guzmán, 2007).

Desde la perspectiva del aprendizaje socioemocional (Alcalay, Berger, Milicic \& Fantuzzi, 2012; Hoffman, 2009; Payton et al., 2007), la relación entre aspectos socioemocionales y académicos se da por diferentes caminos. Perspectivas individuales del desarrollo que se basan en el paradigma de la inteligencia emocional (Mayer \& Salovey, 1997) se centran en el desarrollo de competencias, las cuales tienen impacto directo en el aprendizaje al incluir capacidades de planificación, atención, memoria, y metacognición. También se ha planteado que niveles de bienestar socioemocional positivo generan en el individuo un estado propicio para el aprendizaje, al inhibir pensamientos negativos y autoderogativos (Fernández-Berrocal, Extremera \& Palomera, 2008; Goleman, 2012).

Por otra parte, el aprendizaje socioemocional considera también el contexto en el cual se desenvuelve el individuo; de esta forma, los vínculos que establecen los estudiantes tanto con sus compañeros como con el profesor son centrales para potenciar un ambiente propicio para el aprendizaje, lo cual puede operacionalizarse como un clima escolar positivo y nutritivo (Arón \& Milicic, 1999; Jennings \& Greenberg, 2009; Koplow, 2005; Pianta, La Paro \& Hamre, 2012). Distintos estudios muestran la importancia del clima social escolar para el rendimiento académico (Esposito, 1999; Jia et al., 2009) y para el bienestar de los estudiantes (Cemalcilar, 2010; Kuperminc, Leadbeater, Emmons \& Blatt, 1997; Loukas \& Robinson, 2004). De esta forma, la dimensión socioemocional no solo ha re- cibido atención desde su relación con el desempeño académico, sino también como un objetivo de intervención relacionado con indicadores de salud mental (Alcalay et al., 2012; Durlak et al., 2011; Greenberg, Weissberg \& O'Brien, 2003; Vostanis, Humphrey, Fitzgerald, Deighton \& Wolpert, 2013). Esto ha incrementado los esfuerzos por medir variables socioemocionales, tanto individuales como contextuales. Sin embargo, la integración entre una perspectiva académica (rendimiento) y una de salud mental (bienestar) aún constituye un desafío para investigadores e interventores (Hoffman, 2009).

No obstante lo anterior, modelos actuales de aprendizaje han ido superando perspectivas centradas exclusivamente en habilidades individuales y han puesto el énfasis en dimensiones tanto del individuo, del contexto y de las interacciones entre ambos (Elias \& Haynes, 2008). Por ejemplo, Maquilón Sánchez y Hernández Pina (2011) presentan el modelo 3P desarrollado por Biggs, el cual contempla tres fases que se relacionan de manera sistémica entre ellos: (1) Presagio, que considera las características con las cuales los estudiantes llegan a la situación de aprendizaje y las características previas del contexto educacional; (2) Proceso, que se refiere a la manera en que los estudiantes, en una situación contextual específica, abordan el proceso de enseñanza-aprendizaje y (3) Producto, que constituye el aprendizaje en sí y se operacionaliza como satisfacción con el aprendizaje, rendimiento u otro indicador de logro. Estos estudios han permitido comprobar la relevancia de considerar tanto aspectos individuales como contextuales en el aprendizaje (De la Fuente, Martínez, Peralta \& García, 2010). A pesar de que estos estudios se han llevado a cabo con población universitaria (con todas las particularidades que ello implica) y por lo mismo no son necesariamente transferibles a población de educación básica o de otros contextos socioculturales, han abierto una importante línea de investigación respecto de las distintas variables tanto individuales como de contexto que pueden considerarse (presagio), así como de los procesos que pueden ser relevantes para el aprendizaje.

A nivel individual, la autoestima es uno de los constructos más estudiados (Marsh \& Craven, 
2006; Ross \& Broh, 2000; Trautwein, Lüdtke, Köller \& Baumert, 2006). Sin embargo, la relación entre autoestima y rendimiento no es clara y existe evidencia que cuestiona la direccionalidad de las relaciones establecidas entre ambos (Baumeister, Campbell, Krueger \& Vohs, 2003; Humphrey, 2004; Menon, Tobin, Corby, Menon, Hodges \& Perry, 2007; Peixoto \& Almeida, 2009), y en especial la diferencia entre el reporte de profesores y el propio reporte de los estudiantes (Berger et al., 2011). En este contexto, diversos autores han argumentado acerca de la influencia de profesores y compañeros en la construcción de la autoestima y, consecuentemente, en el rendimiento académico. En específico, la relación entre estudiantes y sus profesores ha sido señalada como un factor clave para un buen desempeño académico y bienestar socioemocional de los estudiantes, actuando a través de las competencias socioemocionales y la motivación del profesorado y su efecto modelador (Frenzel, Goetz, Lüdtke, Pekrun \& Sutton, 2009; Jennings $\&$ Greenberg, 2008; Roth, Assor, Kanat-Maymon \& Kaplan, 2007), sus expectativas y conocimiento respecto de sus estudiantes (Little, 2005) y los vínculos afectivos que establecen (Berger, Milicic, Alcalay, Torretti, Arab \& Justiniano, 2009; Bergin $\&$ Bergin, 2009). Asimismo, los vínculos que los estudiantes establecen con sus pares también constituyen un factor relevante para los logros académicos y de bienestar general (Berger et al., 2009; Erath, Flanagan \& Bierman, 2008; Feldman et al, 2008; Kindermann, 2007; Ryan, 2001). Esto refuerza el hecho de que el aprendizaje socioemocional no puede ser medido únicamente a través de características o competencias individuales, sino también considerando variables sociales y de la relación entre el individuo y su contexto.

En resumen, la evidencia actual está demostrando que existe una relación de influencia entre la dimensión socioemocional y los indicadores académicos, y que las intervenciones que abordan los aspectos socioafectivos tienen un impacto positivo en los resultados en pruebas estandarizadas. Si bien existe creciente evidencia sobre estos aspectos en contextos europeos y Estados Unidos (Pianta \& Allen, 2008; Roeser, Eccles \& Sameroff, 2000), los estudios que abordan esta temática en Latinoamérica, y más específicamente en Chile, son escasos (George, Piña, Flotts, Squicciarini \& Guzmán, 2012; Guzmán et al., 2011)); lo anterior resulta preocupante considerando que la realidad tanto de los estudiantes como de los contextos educacionales en Latinoamérica difiere considerablemente de otros contextos en donde se ha levantado la evidencia señalada. En relación con lo anterior, el presente estudio busca aportar evidencia local que permita comprender la relación entre variables socioemocionales, a nivel individual y de contexto, y el rendimiento académico. Se hipotetizó que por sobre la estabilidad en el rendimiento académico de los estudiantes en el periodo de un semestre, la dimensión socioemocional sería un predictor significativo de cambios en el rendimiento. Específicamente, se hipotetizó que tanto la relación entre estudiantes y profesores así como entre pares (operacionalizadas a través de la percepción de dichas relaciones por los estudiantes), la autoestima de los estudiantes percibida por sus profesores (otro indicador de la relación profesor-estudiante) e indicadores de bienestar emocional estarían asociados a un aumento en el rendimiento académico en el periodo de un semestre.

\section{Método}

\section{Muestra}

Los participantes correspondían a estudiantes de 5.ํ y 6.a año de educación general básica (EGB) pertenecientes a tres establecimientos particulares subvencionados, de nivel socioeconómico medio y medio bajo de Santiago de Chile, los cuales son parte de un estudio mayor orientado al diseño de un programa para favorecer el bienestar y aprendizaje socioemocional. Todos los estudiantes de 5. y $6 .^{\circ}$ EGB de ambos establecimientos fueron invitados a participar. La muestra total incluyó 465 alumnos distribuidos en 13 cursos ( 7 de $5 .^{\circ}$ y 6 de 6.9 ) , distribuidos equitativamente por género (52.1\% mujeres). Para su inclusión en el estudio se solicitó consentimiento informado de los padres, así como asentimiento de los mismos alumnos, 
siguiendo los estándares del Comité de Ética de la universidad patrocinante y de la Comisión $\mathrm{Na}$ cional de Investigación Científica y Tecnológica de Chile (CONICYT). En dicho consentimiento se aseguró la confidencialidad de la información entregada.

Todos los participantes respondieron cuestionarios de autorreporte que evalúan su autoestima, bienestar socioemocional y percepción del clima social escolar. Además, se pidió a los profesores jefes que evaluaran el nivel de autoestima de cada uno de sus estudiantes y se solicitó al establecimiento el reporte del rendimiento académico de los participantes. Los instrumentos de evaluación fueron aplicados colectivamente y en la sala de clases por profesionales y se administraron en el mismo orden a todos los participantes, descartando así efectos diferenciales.

\section{Instrumentos}

\section{Rendimiento Académico}

Se recolectó el promedio general de notas que obtuvieron los estudiantes durante el segundo semestre del año 2011 y el primer semestre del 2012. Este promedio considera todas las asignaturas del currículum nacional y va en una escala de 1 a 7 .

\section{Autoestima}

Se evaluó mediante el Test de Autoestima Escolar ([TAE]; Marchant, Haeussler \& Torretti, 2002). Esta es una prueba de screening o tamizaje que permite conocer el nivel general de autoestima de estudiantes de $3 .^{\circ}$ a $8 .^{\circ}$ año de EGB. Cuenta con 2 versiones complementarias: una del alumno y otra del profesor. La del alumno corresponde a un cuestionario de autorreporte que consta de 23 afirmaciones de respuesta sí o no. La versión del profesor está compuesta por 19 ítemes con 4 alternativas que evalúan frecuencia. El TAE, conformado a partir de una selección de ítems del test Piers Harris, fue estandarizado en Chile y cuenta con normas elaboradas en puntaje $T$ que permiten ubicar el nivel de autoestima del niño en categorías.

\section{Bienestar Socioemocional}

Se utilizó la escala de Autorreporte de Bienestar Socioemocional (ASE) para estudiantes de 4. a 8.. año de enseñanza básica elaborada por Milicic, Arab, Alcalay, Berger y Torretti (ver Arab, 2009). Esta escala de categorías tipo Likert se compone de 52 afirmaciones con cuatro posibilidades de respuesta. El estudiante debe marcar la alternativa que mejor lo describe.

\section{Clima social- escolar}

Fue evaluado utilizando la Escala de Clima Social en Sala de Clases (ECLIS), desarrollada por Arón, Milicic y Armijo (2012). Este instrumento consta de 82 ítems con cuatro alternativas de categorías tipo Likert. Los ítems se organizan en torno a cuatro escalas que indagan en la percepción de los estudiantes respecto a los profesores, compañeros, lugares del establecimiento y la escuela en su globalidad.

\section{Plan de análisis}

Los datos obtenidos fueron consolidados para los procesos estadísticos y desarrollados con el programa SPSS (versión 19). Se condujo análisis de correlación para determinar el grado de asociación entre el rendimiento académico y las variables socioemocionales evaluadas: autoestima, bienestar socioemocional y clima social escolar. Para estudiar esta asociación se aplicó un análisis de regresión lineal por bloques, utilizando método forward (hacia adelante). La variable dependiente fue el rendimiento académico en el primer semestre de 2012. Se incluyó como variables independientes, en el primer bloque, al rendimiento en el período académico anterior (segundo semestre de 2011) y género. En el segundo bloque se integró la autoestima, el bienestar socioemocional y clima social escolar, respectivamente.

\section{Resultados}

En primer lugar, se presentan descriptivos por género para las variables del estudio. Como se observa en 
la Tabla 1 las mujeres presentaron mejor rendimiento académico en ambas mediciones $(t=2.48$ y 3.48 , $p<0.05)$. Asimismo, las mujeres presentaron una mejor percpeción del clima escolar relacionado con los lugares de la escuela $(t=2.41, p<0.05)$. Como puede observarse en la Tabla 2, todas las variables socioemocionales se relacionaron positivamente con rendimiento académico (en sus dos mediciones) así como entre ellas ( $r$ entre 0.1 y $0.89, p<0.01$ ). La única excepción fue la ausencia de asociación observada para la percepción del clima en la relación con los profesores y de la escuela en su globalidad. Los mismos análisis por género mostraron diferencias solo para las asociaciones entre la autoestima reportada por el profesor y la percepción del clima en la relación con los profesores (significativa solo para mujeres), y entre la autoestima reportada por el profesor y la percepción del clima en la relación con los lugares (significativa solo para hombres).

Luego, se realizó un análisis de regresión lineal para observar el efecto de las variables socioemocionales sobre el rendimiento académico en la segunda medición, controlado por el rendimiento académico en la primera medición. En dichos análisis se controló por género, considerando las diferencias observadas. En el paso 1 se incluyó el rendimiento en la primera medición y el género como predictores del rendimiento en la segunda medición. En el paso 2 (método forward) se incluyeron todas las variables socioafectivas estudiadas. Como se observa en la

\section{TABLA 1}

Promedio, desviación estándar y comparaciones por género

\begin{tabular}{lcccc}
\hline & Mujeres & Hombres & $t$ & sig \\
\hline Rendimiento Académico 1 & $5.88(0.48)$ & $5.76(0.52)$ & 2.48 & 0.014 \\
Rendimiento Académico 2 & $5.78(0.53)$ & $5.63(0.58)$ & 3.48 & 0.001 \\
Autoestima Estudiante & $18.78(4.03)$ & $18.8(3.19)$ & $<1$ & n. s. \\
Autoestima Profesor & $57.39(11.2)$ & $56.64(10.77)$ & $<1$ & n. s. \\
Clima-profesores & $3(0.4)$ & $3.01(0.38)$ & $<1$ & n. s. \\
Clima-pares & $2.96(0.51)$ & $3.01(0.5)$ & 1.42 & n. s. \\
Clima-lugares & $3.06(0.48)$ & $2.98(0.51)$ & 2.41 & 0.016 \\
Clima-escuela & $3.19(0.4)$ & $3.15(0.41)$ & 1.3 & n. s. \\
Bienestar Socioemocional & $3.26(0.43)$ & $3.25(0.43)$ & $<1$ & n.s. \\
\hline
\end{tabular}

Fuente: elaboración propia

\section{TABLA 2}

Asociaciones entre rendimiento académico, autoestima (reportada por el estudiante y el profesor), percepción de clima escolar y bienestar socioemocional

\begin{tabular}{lccccccccc}
\hline & 1 & 2 & 3 & 4 & 5 & 6 & 7 & 8 & 9 \\
\hline Rend. Aca. 1 & - & & & & & & & \\
REnd. Aca. 2 & $0.89^{* *}$ & - & & & & & & \\
AE. Estudiante & $0.29^{* *}$ & $0.28^{* *}$ & - & & & & & \\
AE. Profesor & $0.36^{* *}$ & $0.31^{* *}$ & $0.23^{* *}$ & - & & & & \\
Clima-profesores & $0.22^{* *}$ & $0.2^{* *}$ & $0.28^{* *}$ & $0.1^{* *}$ & - & & & \\
Clima-pares & $0.17^{* *}$ & $0.2^{* *}$ & $0.51^{* *}$ & $0.14^{* *}$ & $0.4^{* *}$ & - & & \\
Clima-lugares & $0.17^{* *}$ & $0.18^{* *}$ & $0.29^{* *}$ & $0.1^{* *}$ & $0.54^{* *}$ & $0.39^{* *}$ & - & \\
Clima-escuela & $0.21^{* *}$ & $0.16^{* *}$ & $0.25^{* *}$ & 0.06 & $0.55^{* *}$ & $0.4^{* *}$ & $0.56^{* *}$ & - & \\
Bienestar SE & $0.31^{* *}$ & $0.32^{* *}$ & $0.57^{* *}$ & $0.28^{* *}$ & $0.42^{* *}$ & $0.61^{* *}$ & $0.44^{* *}$ & $0.5^{* *}$ & - \\
\hline
\end{tabular}

Nota. $* * p<0.01$

Rend. Aca. $=$ Rendimiento académico; $\mathrm{AE}=$ Autoestima; Bienestar SE = Bienestar socioemocional

Fuente: elaboración propia 
Tabla 3 el rendimiento académico es altamente estable a lo largo de un semestre $(\beta=0.89, t=38.67$, $p<0.01)$. Al incluir las variables socioafectivas, la percepción del clima escolar respecto de los lugares de la escuela $(\beta=0.09, t=3.76, p<0.01)$ y respecto de la relación con los pares $(\beta=0.06, t=2.64$, $p<0.01$ ) aparecen como predictores significativos del rendimiento académico.

Por último, considerando la inclusión de variables socioafectivas de orden individual (autoestima y bienestar socioemocional) y de contexto (percepción de clima escolar), se realizaron análisis de regresión separados por ambas dimensiones. En la Tabla 4 se observan los resultados para las variables de orden individual (autoestima y bienestar socioemocional) en el panel superior, y para las variables asociadas a la percepción de contexto (clima social escolar) en el panel inferior. Utilizando el mismo procedimiento, se incluyó primero como predictores del rendimiento académico el rendimiento basal y se controló por género. Luego se incluyeron las variables socioafectivas en el paso 2 (método forward). Respecto de las variables individuales, solo el bienestar socioemocional alcanzó niveles significativos en su asociación con el aumento en el rendimiento académico ( $\beta=$ $0.76, t=2.69, p<0.01)$. En relación con la percepción del clima social escolar mostraron asociaciones significativas con el aumento en el rendimiento la percepción de la relación con los pares $(\beta=0.09, t$ $=3.88, p<0.01)$ y de los lugares en la escuela $(\beta=$ $0.06, t=2.45, p<0.01$ ).

\section{Discusión}

La evidencia y los desarrollos teóricos actuales son claros en establecer una asociación entre las dimensiones socioemocionales y académicas (López, 2007), las cuales en una lectura de mayor profundidad establecen también la imposibilidad de separar la dimensión afectiva de la dimensión cognitiva (Izard, 2009). A pesar de este consenso, esta evidencia aún no es integrada consistentemente en las prácticas educativas y en las políticas públicas, considerando aún al aprendizaje socioemocional como secundario en la labor formativa de las instituciones escolares, lo cual se observa por ejemplo en los énfasis en el curriculum formal y en los programas de formación docente (Alcalay et al., 2012). En este contexto, la generación de evidencia empírica que permita, por una parte, resaltar esta asociación y, por otra, informar programas de intervención y desarrollo de políticas públicas, es imprescindible.

El presente estudio contribuye a este objetivo de distintas maneras. En primer lugar, los resultados presentados fortalecen la evidencia existente sobre la asociación entre la dimensión socioemocional y

TABLA 3

Regresión lineal sobre rendimiento académico en la segunda medición, incluyendo rendimiento académico en la primera medición, género y todas las variables socioafectivas

\begin{tabular}{llccccc}
\hline & & B & SE B & $\beta$ & $t$ & $r 2$ \\
\hline Modelo 1 & (Constante) & 5.57 & 1.36 & & $4.09^{* *}$ & \\
& Rendimiento medición 1 & 0.9 & 0.02 & 0.89 & $38.67^{* *}$ & \\
& Género & 0.23 & 0.23 & 0.02 & 1.01 & 0.788 \\
Modelo 2 & Clima-lugares & 0.92 & 0.25 & 0.09 & $3.76^{* *}$ & 0.796 \\
& & & & & & \\
Modelo 3 & Clima-pares & 0.69 & 0.26 & 0.06 & $2.64^{* *}$ & 0.799 \\
Excluidas & AE estudiante & & & -0.01 & $<1$ & \\
& AE profesor & & & 0.02 & $<1$ & \\
& Clima-profesores & & & 0 & $<1$ & \\
& Clima-escuela & & & -0.03 & 1.06 & \\
& Bienestar SE & & & 0 & $<1$ & \\
\hline
\end{tabular}

Nota. $* * p<0.01$

$\mathrm{AE}=$ Autoestima; Bienestar SE $=$ Bienestar socioemocional

Fuente: elaboración propia 


\section{TABLA 4}

Regresión lineal sobre rendimiento académico en la segunda medición, incluyendo rendimiento académico en la primera medición, género y autoestima, y bienestar socioemocional (panel superior) y clima escolar (panel inferior)

\begin{tabular}{llccccc}
\hline & & B & SE B & $\beta$ & $t$ & $r 2$ \\
\hline Modelo 1 & (Constante) & 5.69 & 1.35 & & $4.21^{* *}$ & \\
& Rendimiento medición 1 & 0.89 & 0.02 & 0.89 & $38.85^{* *}$ & \\
& Género & 0.23 & 0.23 & 0.02 & 1.02 & 0.789 \\
& & & & & & \\
Modelo 2 & Bienestar SE & 0.76 & 0.28 & 0.06 & $2.69^{* *}$ & 0.793 \\
& & & & & & \\
Excluidas & AE estudiante & & & 0.01 & $<1$ & \\
& AE profesor & & & 0.03 & 1.14 & \\
& & & & & \\
Modelo 1 & (Constante) & 5.69 & 1.31 & & $4.33^{* *}$ & \\
& Rendimiento medición 1 & 0.89 & 0.02 & 0.89 & $40.01^{* *}$ & \\
& Género & 0.26 & 0.22 & 0.03 & 1.16 & 0.788 \\
Modelo 2 & Clima-pares & 0.94 & 0.24 & 0.09 & $3.88^{* *}$ & 0.795 \\
Modelo 3 & Clima-lugares & 0.63 & 0.26 & 0.06 & $2.45^{* *}$ & 0.798 \\
Excluidas & Clima-profesores & & & 0 & $<1$ & \\
& Clima-escuela & & & -0.03 & -1.25 & \\
\hline
\end{tabular}

Nota. ** $p<0.01$

$\mathrm{AE}=$ Autoestima; Bienestar $\mathrm{SE}=$ Bienestar socioemocional Fuente: elaboración propia

el rendimiento académico. Estos hallazgos convergen con lo encontrado en investigaciones previas (Durlak et al., 2011; Greenberg et al., 2003) y relevan la necesidad de que todos los estudiantes tengan la oportunidad de desarrollar competencias socioemocionales y de formar parte de contextos escolares nutritivos. Específicamente, el presente estudio sugiere que el bienestar socioemocional y la percepción que los estudiantes tienen acerca de las relaciones que se establecen entre compañeros y los espacios escolares, afectan su rendimiento académico. Esta asociación puede explicarse por el hecho que los estudiantes aprenden mejor cuando están felices, confían en sí mismos y se sienten aceptados y valorados por otros, hipótesis que ha sido confirmada por otros autores (Extremera \& Fernández-Berrocal, 2003; Jiménez \& López-Zafra, 2009), planteando incluso que la dimensión socioafectiva puede actuar como factor protector ante condiciones sociales adversas y su potencial impacto en lo académico (Elias \& Haynes, 2008).

Una segunda contribución de este estudio, que constituye un avance por sobre la simple constata- ción de la asociación entre las variables académicas y socioafectivas, es de orden teórico. La propuesta teórica del presente artículo se basa en el concepto de aprendizaje socioemocional, que toma fuerza a partir de los trabajos de Elias (1997), basados en parte en el paradigma de la inteligencia emocional (Mayer \& Salovey, 1997). En una reciente revisión crítica de esta perspectiva, Hoffman (2009) destaca la necesidad de integrar tanto una perspectiva individual como una perspectiva contextual a la noción de aprendizaje socioemocional. En este sentido, su propuesta es superar la noción funcional de competencias socioafectivas, integrándolas dentro de los contextos en los cuales estas son aprendidas y desplegadas. Así, perspectivas individuales de lo socioafectivo (las cuales en este artículo fueron operacionalizadas a través de la autoestima y el bienestar socioemocional) deben ser comprendidas y articuladas con factores de contexto (en este estudio, el clima social escolar), entendiendo que ambas dimensiones son mutuamente dependientes (Berger et al., 2011). En este sentido, la perspectiva del aprendizaje socioemocional permite un abordaje 
simultáneo e integrado tanto del desarrollo individual de estudiantes (y profesores) como también del desarrollo de la comunidad educativa y de la generación de contextos favorecedores del desarrollo integral de quienes participan de ellos (Elias, 1997; Elsknin \& Elsknin, 2003). Los resultados de este estudio fortalecen la perspectiva del aprendizaje socioemocional y constituyen una operacionalización para su abordaje empírico.

Otra importante contribución a nivel teórico se refiere a la causalidad entre las dimensiones socioafectiva y académica. Si bien la estructura longitudinal de los datos del presente artículo hace suponer que son las variables socioafectivas las que impactan el rendimiento académico, los resultados no debieran interpretarse necesariamente en esa dirección. Los resultados obtenidos señalan que el aumento en el rendimiento académico es explicado en parte por las variables socioafectivas que resultaron significativas en este estudio. Sin embargo, para sacar conclusiones definitivas sería necesario conocer líneas de base de dichas variables y contar con un mayor número de mediciones. Como han señalado otros autores, es probable que la asociación entre ambas dimensiones sea bidireccional (Roeser, Eccles \& Sameroff, 1998), considerando que buenos resultados académicos constituyen también un importante factor motivacional y de sensación de competencia y valía.

En relación con lo anterior, parece importante resaltar un riesgo anticipado por Hoffman (2009) respecto del énfasis observado en la actualidad en la implementación de intervenciones en el ámbito socioemocional, entendiéndolas como un medio para mejorar los resultados académicos y no como un fin en sí mismas. En un contexto en que los indicadores académicos son considerados el gold standard de la calidad de la educación, es importante resaltar que dichos indicadores no pueden priorizarse en detrimento de otras dimensiones del desarrollo humano y que son propias de la función formativa de la escuela, como el velar por la salud mental de estudiantes y profesores, favorecer el desarrollo integral, propiciar una educación inclusiva, y favorecer una ciudadanía activa y responsable (Alcalay et al., 2012; Bisquerra, 2000; Chaux, Lleras \& Velásquez, 2004).
Otro aspecto importante de destacar del presente estudio es el de levantar datos y resultados de una población poco estudiada. Si bien existe conocimiento acumulado sobre la asociación entre las dimensiones socioafectiva y académica, dicho conocimiento ha sido generado en estudios desarrollados en países europeos y en Estados Unidos, y no es necesariamente transferible a contextos latinoamericanos. Contar con resultados generados en la realidad latinoamericana, en este caso chilena, es de gran importancia para poder confirmar los modelos teóricos que basan este estudio, pero también para informar estrategias de intervención y la generación de políticas públicas que sean pertinentes a las características propias a estos contextos.

El presente estudio presenta ciertas limitaciones que deben ser consideradas al interpretar los resultados obtenidos. En primer lugar, las variables que pueden utilizarse como indicadores de la dimensión socioemocional son diversas, y las concepciones teóricas sobre la el aprendizaje socioemocional no son unívocas. En este sentido, en futuras investigaciones sería importante considerar otras variables que permitan comprender la relación entre lo afectivo y lo académico desde el desarrollo de competencias socioemocionales (Bisquerra \& Pérez, 2007) y aspectos relacionados con salud mental y psicopatología (Vostanis et al., 2013), entre otros. Asimismo, la percepción de clima social escolar, si bien apunta a la manera en que los sujetos experimentan el contexto escolar, no es necesariamente un indicador objetivo de las condiciones del ambiente; en este sentido, sería interesante considerar otras variables que se orienten a caracterizar el contexto con independencia del reporte de los sujetos, por ejemplo, el estudio de redes sociales (Cotterell, 2007), el reporte de múltiples actores (Gest, 2006) y la consideración de variables institucionales como por ejemplo los estilos de gestión y liderazgo (Gregory et al., 2010). En la misma línea, en este estudio se utilizó el promedio general como indicador del rendimiento académico; futuros estudios podrían utilizar otros diferenciados tales como autoconcepto académico y motivación. Una limitación de orden conceptual se relaciona con la imposibilidad, en el presente estudio, de estudiar la relación entre 
los aspectos socioemocionales y académicos desde una perspectiva de los procesos involucrados en dicha relación (González, Leal, Segovia \& Arancibia, 2012). Futuros estudios debieran considerar diseños longitudinales con más de dos mediciones e hipótesis, que se orienten a la generación de una comprensión sobre cómo estos aspectos se asocian.

Las limitaciones recién señaladas constituyen las futuras líneas de investigación que este estudio abre. Lo anterior es especialmente relevante considerando que la mayor parte de los estudios dentro de esta orientación han sido desarrollados en países europeos y en los Estados Unidos, por lo que los resultados de este estudio permiten ampliar este conocimiento a nuevas poblaciones y sustentar la universalidad de dichas asociaciones. La consideración del aprendizaje socioemocional como una dimensión central a la función formativa de la escuela debe ser enfatizada para favorecer el desarrollo integral de niños y jóvenes, pero también para la generación de espacios escolares nutritivos y una sociedad que valore el bienestar de todos sus miembros.

\section{Referencias}

Alcalay, L., Berger, C., Milicic, N., \& Fantuzzi, X. (2012). Aprendizaje socioemocional y apego escolar: favoreciendo la educación en diversidad. En I. Mena, M. Lissi, L. Alcalay \& N. Milicic (Eds.), Educación y diversidad: aportes desde la psicología educacional (pp. 47-68). Santiago: Ediciones Universidad Católica de Chile.

Arab, M. (2009). Diseño y construcción de escala de autorreporte de aprendizaje socioemocional para niños de 8 a 12 años, de educación general básica. Tesis para optar al grado de Magíster en Psicología Educacional, Pontificia Universidad Católica de Chile.

Arón, A. M., \& Milicic, N. (1999). Clima social escolar y desarrollo personal: un programa de mejoramiento. Santiago: Editorial Andrés Bello.

Arón, A. M., Milicic, N., \& Armijo, I. (2012). Clima social escolar: una escala de evaluación -Escala de Clima Social Escolar, ECLIS-. Universitas Psychologica, 11(3), 803-813.

Baumeister, R., Campbell, J., Krueger, J., \& Vohs, K. (2003). Does high self-esteem cause better per- formance, interpersonal success, happiness, or healthier lifestyles? Psychological Science in the Public Interest, 4(1), 1-44. doi:10.1111/1529-1006.01431

Berger, C., Alcalay, L., Torretti, A., \& Milicic, N. (2011).

Socio-emotional well-being and academic achievement: Evidence from a multilevel approach. Psicologia: Reflexao e Critica, 24(2), 344-351. doi:10.1590/ S0102-79722011000200016

Berger, C., Milicic, N., Alcalay, L., Torretti, A., Arab, M. P., \& Justiniano, B. (2009). Bienestar socioemocional en contextos escolares: la percepción de estudiantes chilenos. Estudios sobre Educación, 17, 21-43.

Bergin, C. A., \& Bergin, D. A. (2009). Attachment in the classroom. Educational Psychology Review, 21(2), 141-170. doi:10.1007/s10648-009-9104-0

Bisquerra, R., \& Pérez, N. (2007). Las competencias emocionales. Educación, 21(10), 61-82.

Bisquerra, R. (2000). Educacional emocional y bienestar. Barcelona: Praxis.

Caso-Niebla, J., \& Hernández-Guzmán, L. (2007). Variables que inciden en el rendimiento académico de adolescentes mexicanos. Revista Latinoamericana de Psicología, 39(3), 487-501.

Cemalcilar, Z. (2010). Schools as socialization contexts: Understanding the impact of school climate factors on students' sense of school belonging. Applied Psychology: An International Review, 59(2), 243-272. doi:10.1111/j.1464-0597.2009.00389.x

Chaux, E., Lleras, J., \& Velásquez, A. (2004). Competencias ciudadanas: de los estándares al aula. Bogotá: Ediciones Uniandes.

Cotterell, J. (2007). Social networks in youth and adolescence. New York: Routledge.

De la Fuente, J., Martínez, J. M., Peralta, F. J., \& García, A. B. (2010). Percepción del proceso de enseñanzaaprendizaje y rendimiento académico en diferentes contextos instruccionales de la Educación Superior. Psicothema, 22(4), 806-812.

Durlak, J., Weissberg, R., Dymnicki, A., Taylor, R., \& Schellinger, K. (2011). The impact of enhancing students' social and emotional learning: A metaanalysis of school-based universal interventions. Child Development, 82(1), 405-432. doi:10.1111/ j.1467-8624.2010.01564.x 
Elias, M. J. (1997). The missing piece: Making the case for greater attention to social and emotional learning. Education Week, 17(5), 36-47.

Elias, M. J., \& Haynes, N. M. (2008). Social competence, social support, and academic achievement in minority, low-income, urban elementary school children. School Psychology Quarterly, 23(4), 474495. doi:10.1037/1045-3830.23.4.474

Elksnin, L. K., \& Elksnin, N. (2003). Fostering social-emotional learning in the classroom. Education, 124(1), 63-48.

Erath, S. A., Flanagan, K. S., \& Bierman, K. B. (2008). Early adolescent school adjustment: Contributions of friendship and peer victimization. Social Development, 17(4), 853-870. doi:10.1111/j.14679507.2008.00458.x

Esposito, C. (1999). Learning in urban blight: School climate and its effect on the school performance of urban, minority, low-income children. School Psychology Review, 28(3), 365-377.

Extremera, N., \& Fernández-Berrocal, P. (2003). La inteligencia emocional en el contexto educativo: hallazgos científicos de sus efectos en el aula. Revista de Educación, 332, 97-116.

Feldman, L., Goncalves, L., Chacón-Puignau, G., Zaragoza, J., Bagés, N., \& De Pablo, J. (2008). Relaciones entre estrés académico, apoyo social, salud mental y rendimiento académico en estudiantes universitarios venezolanos. Universitas Psychologica, 7(3), 739-751.

Fernández-Berrocal, P., Extremera, N., \& Palomera, R. (2008). Emotional intelligence as a crucial mental ability on educational context. En A. Valle \& J. C. Nuñez (Eds.), Handbook of instructional resources and applications in the classroom (pp. 67-88). New York: Nova Science Publishers.

Frenzel, A. C., Goetz, T., Lüdtke, O., Pekrun, R., \& Sutton, R. E. (2009). Emotional transmission in the classroom: Exploring the relationship between teacher and student enjoyment. Journal of Educational Psychology, 101(3), 705-716. doi: 10.1037/ a0014695

George, M., Guzmán, J., Flotts, M., Squicciarini, A. M., \& Guzmán, M. P. (2012). Salud mental en escuelas vulnerables: evaluación del componente promocional de un programa nacional. Revista de Psicología, 21(2), 55-81.

Gest, S. (2006). Teacher reports of children's friendships and social groups: Agreement with peer reports and implications for studying peer similarity. Social Development, 15(2), 248-259. doi:10.1046/j.14679507.2006.00339.x

Goleman, D. (2012). El cerebro y la inteligencia emocional: nuevos descubrimientos. Barcelona: Ediciones B.

González, M. L., Leal, D., Segovia, C., \& Arancibia, V. (2012). Autoconcepto y talento: una relación que favorece el logro académico. Psykhe, 21(1), 37-53. doi:10.4067/S0718-22282012000100003

Greenberg, M., Weissberg, R., \& O’Brien, M. (2003). Enhancing school-based prevention and youth development through coordinated social, emotional and academic learning. American Psychologist, 58(6-7), 466-474. doi:10.1037/0003-066X.58.6-7.466

Gregory, A., Cornell, D., Fan, X., Sheras, P., Shih, T., \& Huang, F. (2010). Authoritative school discipline: High school practices associated with lower bullying and victimization. Journal of Educational Psychology, 102(2), 483-496. doi:10.1037/a0018562

Guzmán, M. P., Jellinek, M., George, M., Hartley, M., Squicciarini, A. M., Canenguez, K., et al. (2011). Mental health matters in elementary school: First grade screening predicts fourth grade achievement test scores. European Child and Adolescent Psychiatry, 20(8), 401-411.

Hoffman, D. (2009). Reflecting on social emotional learning: A critical perspectives on trends in the United States. Review of Educational Research, 79(2), 533-556. doi: 10.3102/0034654308325184

Humphrey, N. (2004). The death of the feel-good factor? Self-esteem in the educational context. School Psychology International, 25(3), 347-360. doi: 10.1177/0143034304046906

Izard, C. (2009). Emotion theory and research: Highlights, unanswered questions, and emerging issues. Annual Review of Psychology, 60, 1-25. doi: 10.1146/ annurev.psych.60.110707.163539

Jennings, P., \& Greenberg, M. (2009). The prosocial classroom: Teacher social and emotional competence in relation to student and classroom outcomes. Review of Educational Research, 79(1), 491525. doi:10.3102/0034654308325693 
Jia, Y., Way, N., Ling, G., Yoshikawa, H., Chen, X., Hughes, D., Ke, X., et al. (2009). The influence of student perceptions of school climate on socioemotional and academic adjustment: A comparison of Chinese and American adolescents. Child Development, 80(5), 1514-1530. doi:10.1111/j.14678624.2009.01348.x

Jiménez, M. I., \& López-Zafra, E. (2009). Inteligencia emocional y rendimiento escolar: estado actual de la cuestión. Revista Latinoamericana de Psicología, 41(1), 69-79.

Kindermann, T. A. (2007). Effects of naturally-existing peer groups on changes in academic engagement in a cohort of sixth graders. Child Development, 78(4), 1186-1203. doi:10.1111/j.1467-8624.2007.01060.x

Koplow, L. (2005). Escuelas que curan: la construcción de climas emocionalmente saludables. Buenos Aires: Troquel.

Kuperminc, G., Leadbeater, B., Emmons, C., \& Blatt, S. (1997). Perceived school climate and difficulties in the social adjustment of middle school students. Applied Developmental Science, 1(2), 76-88.

Little, E. (2005). Secondary school teachers' perceptions of students' problem behaviours. Educational Psychology, 25(4), 369-377. doi:10.1080/01443410500041516

López, V. (2007). La inteligencia social: aportes desde su estudio en niños y adolescentes con altas capacidades cognitivas. Psykhe, 16(2), 17-28. doi:10.4067/ S0718-22282007000200002

Loukas, A., \& Robinson, S. (2004). Examining the moderating role of perceived school climate in early adolescent adjustment. Journal of Research on Adolescence, 14(2), 209-233. doi:10.1111/j.15327795.2004.01402004.x

Maquilón Sánchez, J., \& Hernández Pina, F. (2011). Influencia de la motivación en el rendimiento académico de los estudiantes de formación profesional. REIFOP, 14(1), 81-100.

Marchant, T., Haeussler, I., \& Torretti, A. (2002). Batería de Test de Autoestima Escolar. Santiago, Chile: Ediciones Universidad Católica de Chile.

Marsh, H., \& Craven, R. (2006). Reciprocal effects of self-concept and performance from a multidimensional perspective: Beyond seductive pleasure and unidimensional perspectives. Perspectives on Psy- chological Science, 1(2), 133-163. doi:10.1111/j.17456916.2006.00010.x

Mayer, J. D., \& Salovey, P. (1997). What is emotional intelligence? In P. Salovey \& D. Sluyter (Eds), Emotional development and emotional intelligence: Implications for educators (pp. 3-31). New York: Basic Books.

Menon, M., Tobin, D. D., Corby, B. C., Menon, M., Hodges, E. V. E., \& Perry, D. G. (2007). The developmental costs of high self-esteem for antisocial children. Child Development, 78(6), 1627-1639. doi:10.1111/j.1467-8624.2007.01089.x

Payton, J., Weissberg, R., Durlak, J., Dymnicki, A., Taylor, R., Schellinger, K., \& Pachan, M. (2007). Positive impact of social and emotional learning for kindergarten to eighth-grade students: Findings from three scientific reviews. Chicago: Collaborative for Academic, Social, and Emotional Learning. Recuperado de http://casel.org/wp-content/uploads/ PackardTR.pdf

Peixoto, F., \& Almeida, L. (2009). Self-concept, selfesteem and academic achievement: Strategies for maintaining self-esteem in students experiencing academic failure. European Journal of Psychology of Education, 25(2), 157-175. doi:10.1007/s10212010-0011-z

Pianta, R., \& Allen, J. P. (2008). Building capacity for positive youth development in secondary school classrooms: Changing teachers' interactions with students. In M. Shinn \& H. Yoshikawa (Eds.), Towards positive youth development: Transforming schools and community programs (pp. 21-39). New York: Oxford University Press.

Pianta, R., La Paro, K., \& Hamre, B. (2012). Classroom assessment scoring system. Baltimore, MD: Paul H. Brookes Publishing.

Roeser, R., Eccles, J., \& Sameroff, A. (1998). Academic and emotional functioning in early adolescence: Longitudinal relations, patterns, and prediction by experience in middle school. Development and Psychopathology, 10(2), 321-352.

Roeser, R., Eccles, J., \& Sameroff, A. (2000). School as a context of early adolescents' academic and social-emotional development: A summary of research findings. The Elementary School Journal, 100(5), 443-471. 
Ross, C., \& Broh, B. (2000). The roles of self-esteem and the sense of personal control in the academicachievement process. Sociology of Education, 73(4), 270-284.

Roth, G., Assor, A., Kanat-Maymon, Y., \& Kaplan, H. (2007). Autonomous motivation for teaching: How self-determined teaching may lead to self-determined learning. Journal of Educational Psychology, 99(4), 761-774. doi:10.1037/0022-0663.99.4.761

Ryan, A. (2001). The peer group as a context for the development of young adolescent motivation and achievement. Child Development, 72(4), 1135-1150. doi: $10.1111 / 1467-8624.00338$
Trautwein, U., Lüdtke, O., Köller, O., \& Baumert, J. (2006). Self-esteem, academic self-concept, and achievement: How the learning environment moderates the dynamics of self-concept. Journal of Personality and Social Psychology, 90(2), 334-349. doi: 10.1037/0022-3514.90.2.334

Vostanis, P., Humphrey, N., Fitzgerald, N., Deighton, J., $\&$ Wolpert, M. (2013). How do schools promote emotional well-being among their pupils? Findings from a national scoping survey of mental health provision in English Schools. Child and Adolescent Mental Health, 18(3), 151-157. doi: 10.1111/j.14753588.2012.00677.x 